\title{
ISOLATION AND GROSS STRUCTURE OF A NEW ANTIBIOTIC SS-56 C AND RELATED COMPOUNDS
}

\author{
Shigeharu Inouye, Takashi Shomura, Hiroshi Watanabe, \\ Kunikazu Totsugawa and Taro NidDa \\ Central Research Laboratories, Meiji Seika Kaisha, Ltd. \\ Kohoku-ku, Yokohama, 222 Japan \\ (Received for publication April 18, 1973)
}

\begin{abstract}
A new antibiotic, designated SS-56 C, has been isolated from a culture broth of Streptomyces eurocidicus No. SS-56. The antibiotic is active against a number of Gram-positive, negative bacteria and fungi. Structural studies including analysis of NMR and in particular MS spectra and acid degradation suggested that SS-56 C substance is the 2-hydroxy derivative of A-396-I substance. Together with SS-56 C substance, were isolated two new hexoglycosides of 2-deoxystreptamine (SS-56 A and B), as well as A-396-I substance (SS-56 D), and gross structures were deduced.
\end{abstract}

Destomycin A (1) and hygromycin B (2) $)^{2}$ are members of the aminocyclitol antibiotics which consist of a diaminocyclitol, neutral sugar and polyhydroxyamino acid. They show broad bioactivity against bacteria and fungi, and are of practical importance against helminths including ascarids in swine. Recently, A-396-I substance $(3)^{3)}$ has been shown to belong to this class of antibiotics containing 2-deoxystreptamine in place of hyosamine ${ }^{4)}$.

In this communication, we report on the isolation of a fourth member of the destomycin group, that is SS-56 C substance (4) of Streptomyces origin.

\section{Production and Isolation of SS-56}

$A, B, C$ and $D^{*}$

A Streptomyces strain No. SS-56 was isolated from a soil collected at Shimonoseki, Yamaguchi Prefecture, Japan and classified as Streptomyces eurocidicus ${ }^{5)}$ from the morphological, cultural and physiological characteristics of the strain.

The SS-56 strain was incubated on a

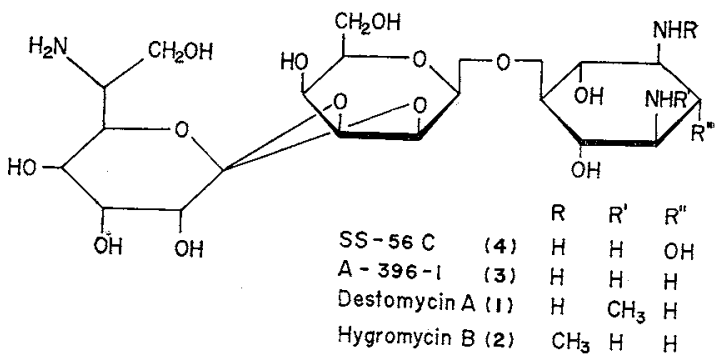

Chart 1. Structures of SS-56 C and related antibiotics tube shaker in a medium consisting of starch $1.0 \%$ and soybean meal $3.0 \%$ (pH 7.0) for 2 days, and then on a recipro shaker in the same medium for 2 days. The culture was transferred into a 50 liter jar fermentor in a medium (35 liters) containing starch $2.0 \%$, soybean meal $2.5 \%$, wheat embryo $1.0 \%$ and $\mathrm{NaCl} 0.25 \%$ (pH 7.0). Fermentation was carried out under aeration at $28^{\circ} \mathrm{C}$, and the production of antibiotics was followed by the bioassay using Streptomyces No. 80 strain as test organism. The maximum titer was reached after 73-hour cultivation.

* The following abbreviations were used. DSA: deoxystreptamine, GLC: gas-liquid chromatography, IR: infrared absorption, MS: mass spectrometry, NMR: nuclear magnetic resonance spectroscopy, RT: retention time, TMS: trimethylsilyl, TLC: thinlayer chromatography, UV: ultraviolet absorption. 
Isolation procedure of SS-56 substances from the broth filtrate was summarized in Scheme 1 . The four components of SS-56 substances were named SS-56 A,B (bioinactive) and C, D (bioactive) in the order of elution from Dowex 1 resin column. SS-56 A and B were crystallized from wateralcohol, while SS-56 C and D were obtained as amorphous powder.

Scheme 1. Isolation procedure of SS-56 substances

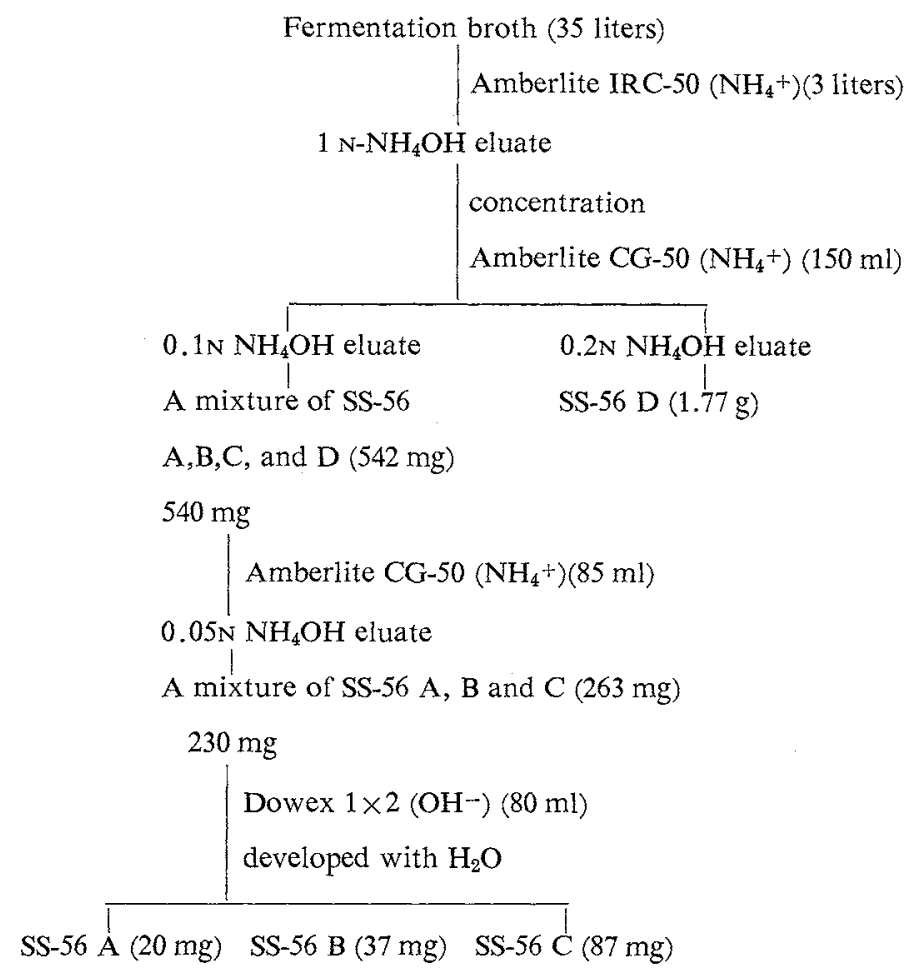

\section{Properties of SS-56 A, B, C and D}

Properties of SS-56 substances were summarized in Table 1. The IR spectrum of SS-56 C is illustrated in Fig. 1.

$\mathrm{Rf}$ values on silica-gel TLC and relative RT of the TMS-derivatives on GLC are shown in Tables 1 and 2. When compared the Rf and the RT of SS-56 substances with those of the known related antibiotics, SS-56 A, B, C substances were differentiated from destomycin A, hygromycin $B$ and A-396-I, indicating that they are novel antibiotics. However, the mobility of SS-56 D, another bioactive ingredient, coincided with that of A-396-I. Identification of SS-56 D with A-396I was verified by the physico-chemical properties shown in Table 1, and MS of $\mathrm{N}$-salicylidene and $\mathrm{N}$-salicylidene-O-TMS-derivatives described later.

Tables 3 and 4 show the antimicrobial spectrum of SS-56 C, which was very close to those reported for the destomycin group. It was noted that SS-56 C showed weaker activity than SS-56 D (A-396-I) against bacteria, but comparable or greater activity against fungi.

Physico-chemical properties of SS-56 A and B that are devoid of bioactivity were similar to those of SS-56 C and D with respects to solubility, color reactions, UV and IR spectra, but opposite in optical activity. 
Table 1. Physico-chemical properties of SS-56 A, B, C, D and A-396-I

\begin{tabular}{|c|c|c|c|c|c|c|c|}
\hline & & & SS-56 A & SS-56 B & SS-56 C & SS-56 D & A-396-I3) \\
\hline & ee $b c$ & & crystalline & crystalline & amorphous & amorphous & amorphous \\
\hline & p. $\left(^{\circ}\right.$ & & $252 \sim 254(\mathrm{dec})$ & $229 \sim 231(\mathrm{dec})$ & $201 \sim 203$ (dec) & $185 \sim 190(\mathrm{dec})$ & $185 \sim 195(\mathrm{dec})$ \\
\hline & ${ }_{0}^{25}(\mathrm{H}$ & & $-30^{\circ}(c 0.62)$ & $-11.5^{\circ}\left(\begin{array}{cc}c & 0.93\end{array}\right)$ & $+12^{\circ}(c 1.08)$ & $+12.7^{\circ}(c 1.02)$ & $+11.6^{\circ}(c 0.936)$ \\
\hline & $\mathrm{D}$ & $\left.{ }_{2} \mathrm{O}\right)$ & plain negative & plain negative: & plain positive & plain positive & \\
\hline & $(\mathrm{H}$ & & end & end & end & end & end \\
\hline & 1. $\mathrm{Y}$ & & $324 *_{1}$ & $324 *_{1}$ & $529 *_{1}$ & $513 *_{1}$ & 513 \\
\hline & 1. fo & mula & $\mathrm{C}_{12} \mathrm{H}_{24} \mathrm{~N}_{2} \mathrm{O}_{8}$ & $\mathrm{C}_{12} \mathrm{H}_{24} \mathrm{~N}_{2} \mathrm{O}_{8}$ & $\mathrm{C}_{19} \mathrm{H}_{35} \mathrm{~N}_{3} \mathrm{O}_{14}$ & $\mathrm{C}_{19} \mathrm{H}_{35} \mathrm{~N}_{3} \mathrm{O}_{13}$ & $\mathrm{C}_{19} \mathrm{H}_{35} \mathrm{~N}_{3} \mathrm{O}_{13}$ \\
\hline & ت & $\mathrm{C}(\%)$ & 44.0 & 43.8 & 42.6 & 44.2 & 45.42 \\
\hline & 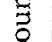 & $\mathrm{H}(\%)$ & 7.6 & 7.7 & 7.1 & 7.0 & 7.07 \\
\hline$\frac{n}{n}$ & I & $\mathrm{N}(\%)$ & 8.4 & 8.3 & 7.6 & 7.9 & 7.93 \\
\hline$\stackrel{\widetilde{g}}{g}$ & $\sigma^{-}$ & $\mathrm{C}(\%)$ & 44.4 & 44.4 & 43.1 & 44.4 & 44.46 \\
\hline & $\frac{\tilde{U}}{\pi}$ & $\mathrm{H}(\%)$ & 7.5 & 7.5 & 6.7 & 6.9 & 6.82 \\
\hline & 0 & $N(\%)$ & 8.6 & 8.6 & 7.9 & 8.2 & 8.18 \\
\hline & $f(T)$ & C) $*_{2}$ & 0.56 & 0.48 & 0.56 & 0.43 & 0.43 \\
\hline & $\mathrm{T}(\mathrm{C}$ & (C) & $\mathrm{RT}_{\mathrm{neamine}} 0.97$ & $R T_{\text {neamine }} 1.05$ & $\mathrm{RT}_{\text {desto. }} 1.10$ & $\mathrm{RT}_{\text {desto.A }} 1.03$ & \\
\hline . & & hydrin & + & + & + & + & + \\
\hline $5:$ & & hrone & + & + & + & + & \\
\hline$\dot{0}^{\circ}$ & & ling & - & - & - & - & - \\
\hline & . & ity* $*_{3}$ & inactive & inactive & active & active & active \\
\hline
\end{tabular}

$*_{1}$ Determined by MS of the $\mathrm{N}$-salicylidene derivatives.

* ${ }_{2}$ Silica-gel TLC developed with $\mathrm{CHCl}_{3}-\mathrm{MeOH}-4 \% \mathrm{NH}_{4} \mathrm{OH}(12: 1: 1)$

$*_{3}$ Paper disc assay against Gram-positive and negative bacteria $(1,000 \mu \mathrm{g} / \mathrm{ml})$.

Fig. 1. IR spectrum of SS-56 C (4) in $\mathrm{KBr}$

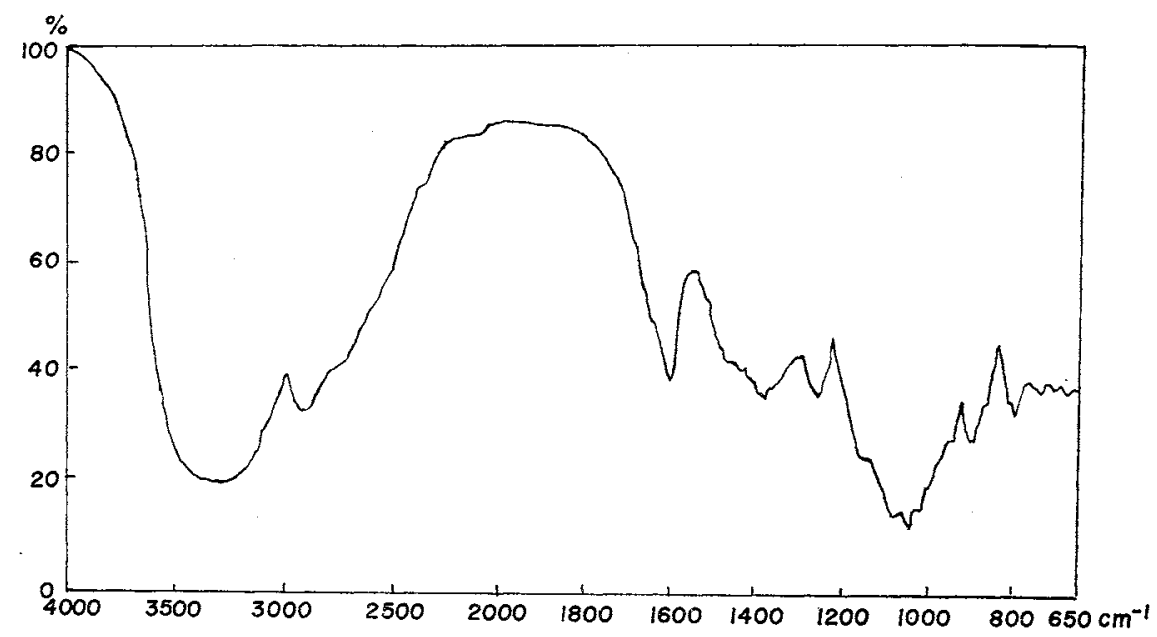

Table 2. Comparison of Rf values of SS-56 C and D with those of destomycin A, hygromycin B and A-396-I on silica-gel TLC*

\begin{tabular}{c|c|c|c|c|c}
\hline Solvent system & SS-56C & SS-56D & Destomycin A & Hygromycin B & A-396-I \\
\hline $\mathrm{CHCl}_{3}-\mathrm{MeOH}-4 \% \mathrm{NH}_{4} \mathrm{OH}(2: 1: 1)$ & 0.47 & 0.35 & 0.27 & 0.30 & 0.35 \\
$10 \% \mathrm{NH}_{4} \mathrm{AcO}-\mathrm{MeOH}(1: 1)$ & 0.69 & 0.68 & 0.51 & 0.53 & 0.68 \\
\hline
\end{tabular}

* Spots were detected by spraying ninhydrin solution. 
Fig. 2. NMR spectra of SS-56 C (4) and A-396-I (3) in $\mathrm{D}_{2} \mathrm{O}$ at $70^{\circ} \mathrm{C}$

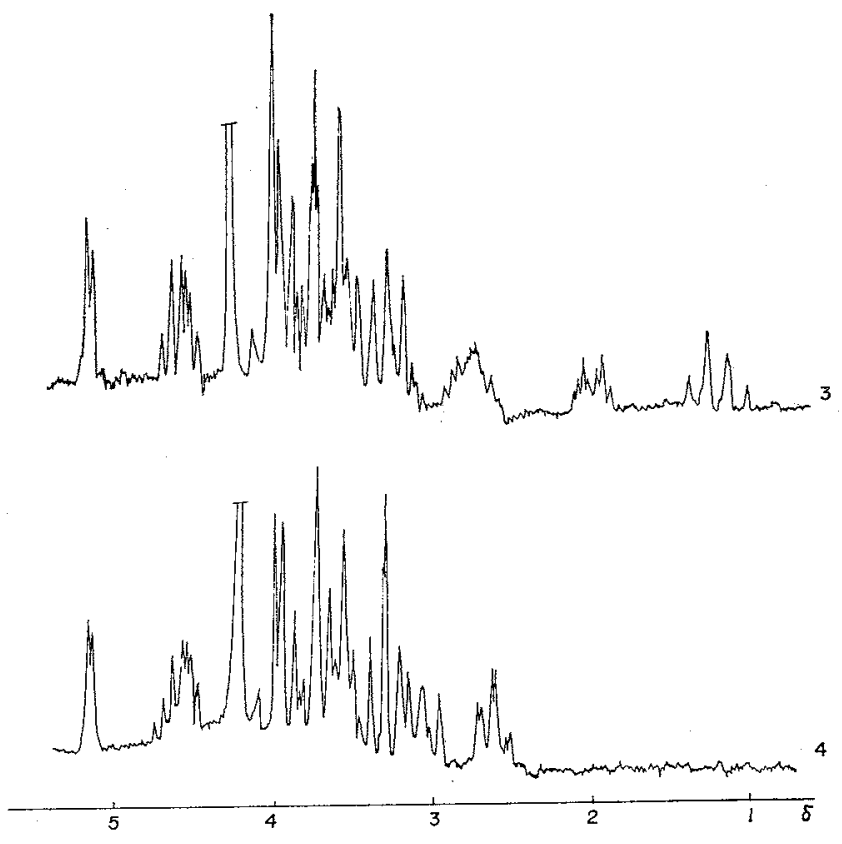

\section{Gross Structure of SS-56 C}

The gross structure of a new antibiotic SS-56 C was examined by utilizing, as model compounds, A-396-I and destomycin A, whose structural features have already been established. Comparison of the NMR spectra of A-396-I (SS-56 D) (3) and SS-56 C (4) in Fig. 2 clearly indicated that the spectrum of 4 lacked in two characteristic methylene signals of 2-DSA and N-methyl signal typical

Table 3. Antimicrobial spectra of SS-56 C and D (A-396-I)

\begin{tabular}{l|c|c}
\multicolumn{1}{c|}{ Test organisms } & \multicolumn{2}{c}{ MIC $(\mu \mathrm{g} / \mathrm{ml})$} \\
\cline { 2 - 3 } & SS-56C & SS-56 D(A-396-1) \\
\hline Bacillus subtilis PCI 219 & 3.125 & 1.56 \\
Escherichia coli & 100 & 50 \\
Klebsiella pneumoniae & 50 & 25 \\
Mycobacterium smegmatis 607 & 25 & 25 \\
$\quad, \quad$ Km-resistant & 100 & 50 \\
" St-resistant & 100 & 100 \\
Proteus vulgaris & 100 & 100 \\
Pseldomonas aeruginosa & 50 & 25 \\
Sarcina lutea & 25 & 12.5 \\
Staphylococcus aureus FDA 209P & 50 & 25 \\
Streptomyces strain, No. 80 & 0.39 & 0.39 \\
Ascochyta sojaecola ABRAMOFF As-11 & 0.97 & \\
Botrytis cinerea PERS & 12.5 & \\
Cladosporium lerbarum LINK & 3.1 & \\
Fusarium oxysporium (F. melonis) & 12.5 & \\
Pellicularia sasaki & 12.5 & \\
Sclerotium bataticola TAUB SB-4 & 3.1 & \\
\hline
\end{tabular}


Table 4. Antifungal spectra of SS-56 C and SS-56 D (A-396-I) assayed by the paper disc method (8 cm)

\begin{tabular}{l|c|c|c|c}
\hline \multirow{2}{*}{ Fungus } & \multicolumn{2}{c|}{ SS-56 C } & \multicolumn{2}{c}{ SS-56 D (A-396-1) } \\
\cline { 2 - 3 } & $800 \mu \mathrm{g} / \mathrm{ml}$ & $400 \mu \mathrm{g} / \mathrm{ml}$ & $800 \mu \mathrm{g} / \mathrm{ml}$ & $400 \mu \mathrm{g} / \mathrm{ml}$ \\
\hline Alternaria tenuis & $(16.7)^{*}$ & $(13.8)$ & $(13.8)$ & $(12.6)$ \\
Ascochyta sojaecala & 22.8 & 18.9 & 21.2 & 16.0 \\
Aspergillus oryzae & 0 & 0 & 0 & 0 \\
Botrytis cinerea & 15.2 & 12.0 & 10.5 & trace \\
Candida krusei & 0 & 0 & 0 & 0 \\
Cladosporium herbarum & 19.0 & 14.3 & 16.7 & 12.4 \\
Cryptococcus neoformans & 17.0 & 14.8 & 16.8 & 14.3 \\
Fusarium oxysporum & $(22.8)$ & $(19.8)$ & $(20.4)$ & $(16.3)$ \\
Mucor spinescens & trace & 0 & trace & 0 \\
Pellicularia sasaki & 20.0 & $(14.2)$ & $(17.0)$ & $(13.6)$ \\
Pyricularia oryzae & 0 & 0 & 0 & 0 \\
Saccharomyces cerevisiae & 11.1 & 10.4 & 11.6 & trace \\
Sclerotium bataticola & 31.2 & 29.8 & 30.9 & 25.6 \\
Torula utilis & 0 & 0 & 0 & 0 \\
Trichophyton asteroides & $(15.0)$ & $(12.8)$ & $(13.8)$ & $(10.5)$ \\
\hline
\end{tabular}

*Parenthesis indicates a faint inhibition zone.

for destomycin A. However, an anomeric proton of $\beta$-D-taloside at 5.27 with $J_{1,2} 2.0 \mathrm{~Hz}$ in 3 was observed as such in 4 at 5.27 with $J_{1,2} 2.0 \mathrm{~Hz}$. Likewise, a multiplet around 4.6 assignable to the methine protons of destomic acid or the deshielded protons due to substitution of destomic acid* was observed in both spectra. Therefore, the difference between SS-56 C and A-396-I was considered to be in the diaminocyclitol moiety, but not in the talose and destomic acid moieties. This assumption was fully supported by the detailed MS analysis, which was in particular a suitable tool for the structural examination in view of the low yield of SS-56 C.

It has been reported that MS of $\mathrm{N}$-salicylidene aminoglycoside antibiotics gave rise to intense molecular ion peaks and simplified fragmentation pattern. ${ }^{6)}$ This technique was now applied to the MS analysis of the destomycin group.

Chart 2 shows the main fragmentation of N-salicylidene A-396-I (SS-56 D) (3a) and SS-56 C (4a). The $\mathrm{M}^{+}$of $\mathbf{4 a}$ was observed at $m / e$ 841, and the $\mathrm{N}$-salicylidene hexosyl-diaminocyclitol ions at $m / e$ 548, 530 and 444. Elemental compositions of these ions revealed that the ions from $4 a$ contained an extra oxygen atom as compared to the corresponding ions from 3a. Likewise, the increase of 16 mass units was observed in the strong di-N-salicylideneiminocyclitol ions appeared at $m / e$ 386, 332 and 282. On the contrary, the N-salicylidene destomic acid-hexose-ester ions were observed at $m / e 455$ and 424 , with no mass shift and no change of elemental composition in $3 a$ and 4a.

These results indicated that the difference between SS-56 C and A-396-I existed in the diaminocyclitol portion, which must be diaminotetrahydroxycyclohexane in SS-56 C. This conclusion was further substantiated by MS analysis of the O-TMS derivatives of N-salicylidene SCHIFF bases (1) $\mathbf{3}, \mathbf{3 b}$, 4b) shown in Chart 3.

* This multiplet was not observed in the spectra of SS-56 A and B that are devoid of destomic acid as shown in Fig. 6. 
Chart 2. MS fragmentation of N-salicylidene SS-56 C (4a) and N-salicylidene A-396-I (3a) *The base peak was arbitrarily selected from the peaks above $m / e 200$

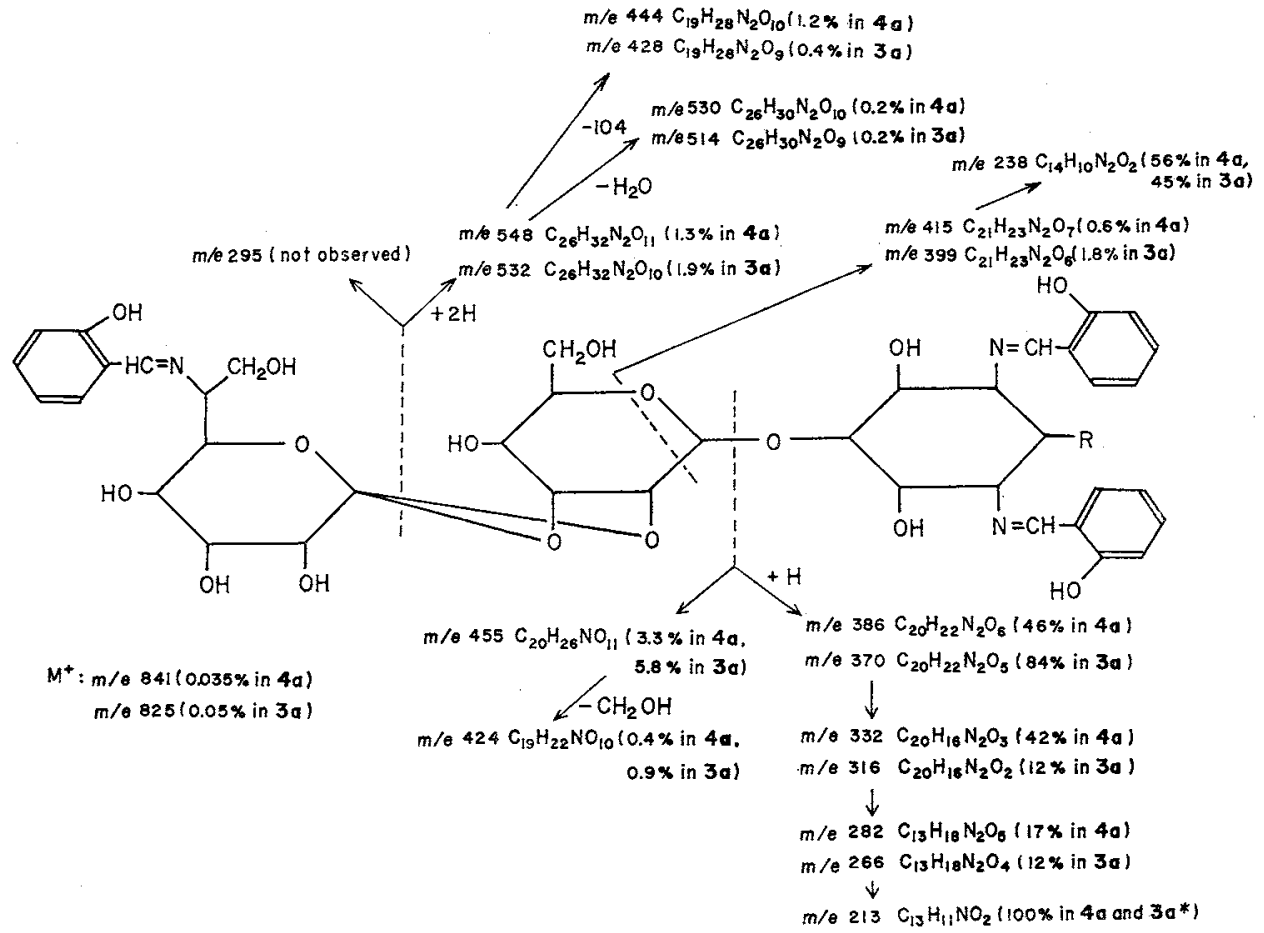

Chart 3. MS fragmentation of N-salicylidene -O-TMS derivatives of SS-56 C (4b), A-396-I (3b) and destomycin A (1b)

*The base peak was arbitrarily selected from the peaks above $m / e 200$

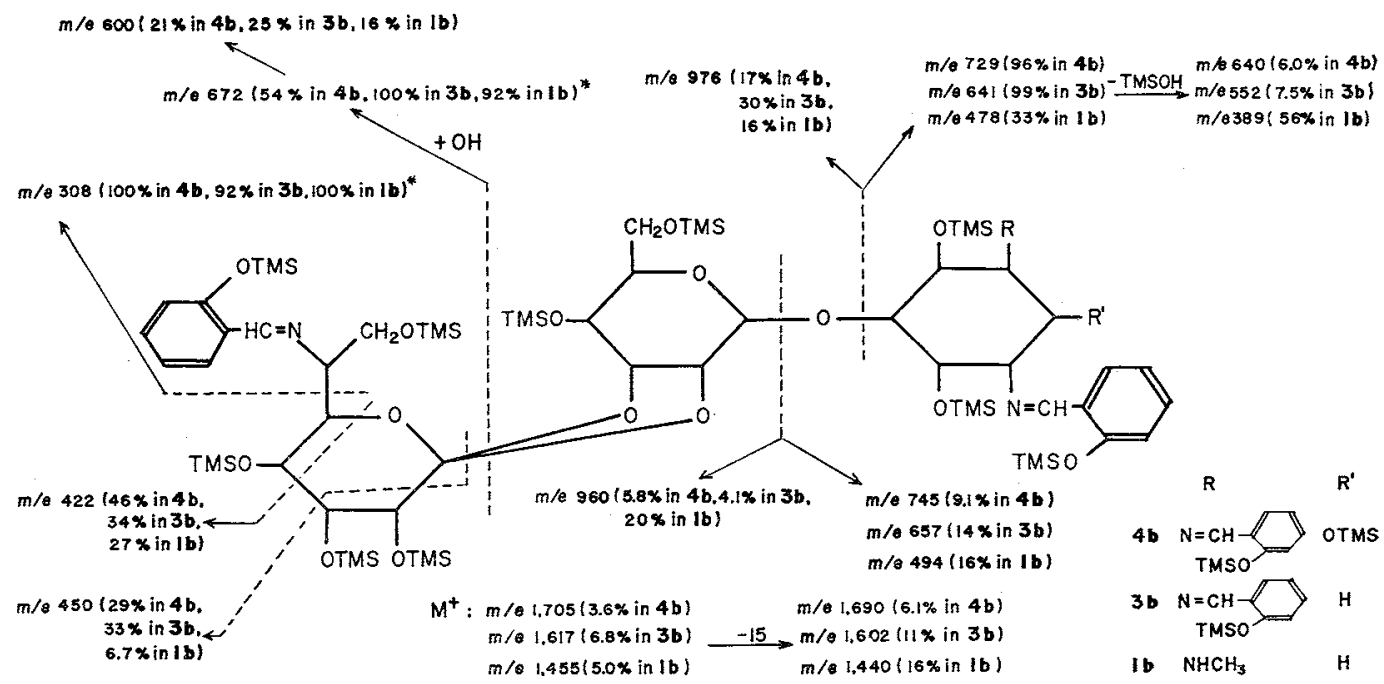

Fragmentation of N-salicylidene-O-TMS-SS-56 C (4b) was in close parallel to those of $\mathbf{1 b}$ and $\mathbf{3 b}$, reflecting the structural similarity of three antibiotics. The $\mathrm{M}^{+}$at $m / e 1,705$, the (M minus methyl $)^{+}$at $m / e 1,690$, and the ions containing diaminocyclitol at $m / e 745,729,639$ all were 
88 units (OTMS) larger than those from 3b. On the other hand, fragment ions from the hexose and destomic acid moieties at $m / e$ 976, 960, 672, 600, 450, 422 and 308 were present with comparable abundance in three antibiotics, suggesting that two of the three structural units were identical in them. Based on the results obtained above, it seemed reasonable to assume that SS-56 $\mathrm{C}$ is the 2-hydroxy derivative of A-396-I. Degradation study summarized in Scheme 2 indicated that diaminocyclitol in $\mathbf{4}$ was in fact streptamine.

The methanolyzate of 4 showed in the IR spectrum two carbonyl bands at 1,780 (weak) and $1,730 \mathrm{~cm}^{-1}$, which, as found in the methanolyzate of 3 , could be assigned to $\gamma$-lactone and methyl ester of destomic acid. On TLC and GLC, the mobilities of the two structural units (methyl taloside and destomic acid ester) liberated from 4 were indistinguishable from those from 3 and $\mathbf{1}$, but diaminocyclitol was differentiated from 2-DSA, hyosamine and 2-epi-streptamine, in particular by GLC (see Table 5). Identification of the diaminocyclitol from 4 with streptamine was first suggested by the identical RT on GLC, and verified by the isolation of crystalline sulfate, whose IR spectrum was superimposable to that of streptamine sulfate prepared from streptomycin.

The same conclusion was derived from the inspection of the hydrolyzate of 4 . The chromatograms of TLC and GLC were illustrated in Figs. 3 and 5, respectively. Again, talose and destomic acid were detected in the hydrolyzate of 4, but neither 2-DSA nor hyosamine could be detected. Instead, a diaminocyclitol corresponded to streptamine was recognized.

At present, we have no definite evidence on the steric mode of linkage of the three structural units in $\mathbf{4}$, but the close resemblance of 3 and $\mathbf{4}$ in their NMR and MS spectra, optical and biological activities as well as simultaneous production suggested the structure 4 shown in Chart 1 . To the best of our knowledge, SS-56 C is the first example of streptamine glycoside found in nature.

\section{Gross Structures of SS-56 A (5) and B (6)}

The IR spectra of 5 and 6 as well as the properties in Table 1 were indicative of polyhydroxypolyamino compounds. The MS spectra of N-salicylidene derivatives of SS-56 A and B (5a and 6a)

Scheme 2. Chemical degradation of SS-56 substances

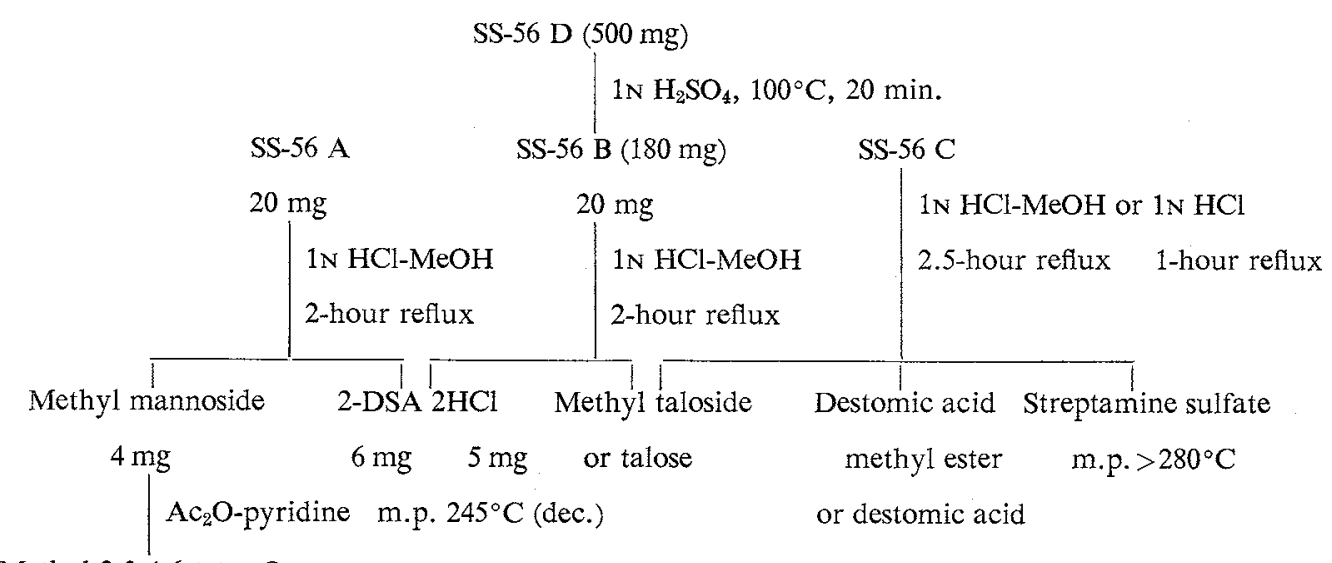

Methyl 2,3,4,6-tetra-O-

acetyl $\alpha$-mannopyranoside

m.p. $59 \sim 60^{\circ} \mathrm{C}$ 
Chart 4. MS fragmentation of N-salicylidene SS-56 A (5a) and B (6a) *The base peak was arbitrarily selected from the peaks above $m / e 200$ **A doublet with stronger $\mathrm{C}_{9} \mathrm{H}_{9} \mathrm{NO}_{2}$ ion

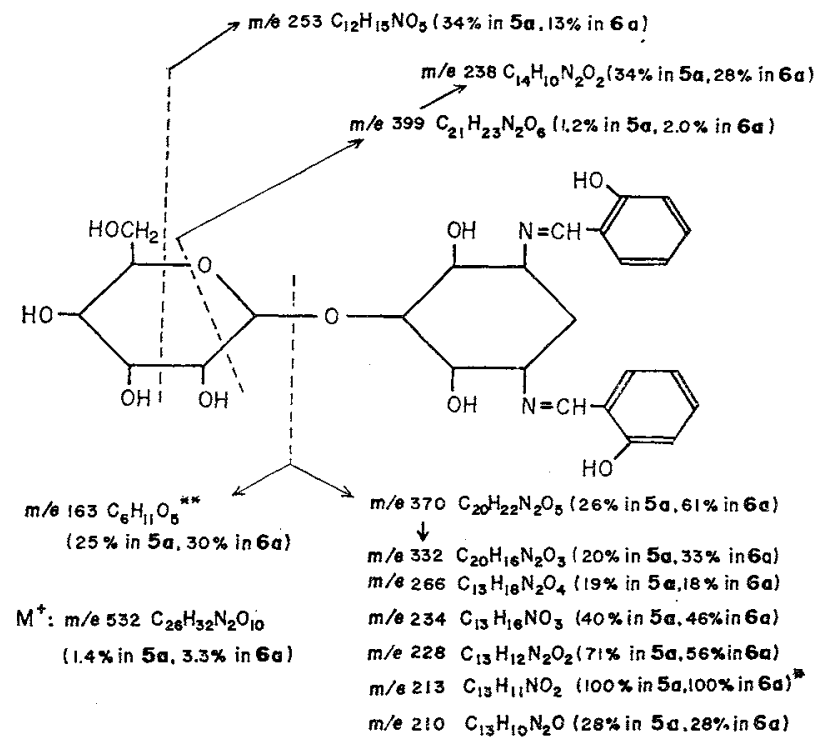

Table 5. Relative RT of methanolyzates and hydrolyzates of SS-56 A, B, C, D (A-396-I) and destomycin A on GLC

\begin{tabular}{|c|c|c|c|}
\hline Methanolyzates*1 & Methyl hexoside & Diaminocyclitol*2 & Destomic ester \& lactone \\
\hline SS-56 A & $0.63 * 3$ & 0.95 & \\
\hline SS-56 B & 0.70 & 0.95 & \\
\hline SS-56 C & 0.70 & 2.0 & $2.2,2.9$ (minor), 3.3 \\
\hline SS-56 D (A-396-I) & 0.70 & 0.95 & $2.2,2.9 \quad(\prime \prime)$ \\
\hline Destomycin A & 0.70 & 0.95 & $2.2,2.9 \quad$ (II) \\
\hline
\end{tabular}

\begin{tabular}{l|c|c|c}
\hline Hydrolyzates*4 & Hexose & Diaminocyclitol & Destomic acid \\
\hline SS-56 C & $0.84,1.05$ (minor) & 1.9 & 2.0 \\
SS-56 D (A-396-1) & $0.84,1.05(" \prime)$ & 0.84 & 2.0 \\
Destomycin A & $0.86,1.05(")$ & 0.86 & 2.0 \\
\hline
\end{tabular}

$*_{1}$ RT of TMS-methyl $\alpha$-D-glucopyranoside was selected as 1.00 .

$*_{2}$ Relative RT of 2-DSA, streptamine and 2-epi-streptamine were 0.95, 2.0 and 1.8, respectively.

$*_{3} \quad$ Relative RT of TMS-methyl $\alpha$-D-mannopyranoside was 0.63 .

$*_{4}$ RT of TMS-D-glucose was arbitrarily selected as 1.00 .

were essentially identical, both showing the $\mathrm{M}^{+}$at m/e 532 with elemental composition $\mathrm{C}_{26} \mathrm{H}_{82} \mathrm{~N}_{2} \mathrm{O}_{10}$. Subtraction of two salicylidene chromophores from the $\mathrm{M}^{+}$gave the same molecular formula $\mathrm{C}_{12} \mathrm{H}_{18} \mathrm{~N}_{2} \mathrm{O}_{8}$ (324) for 5 and 6. As shown in Chart 5, fragmentation of the $\mathrm{M}^{+}$afforded a strong $m / e 370$ peak with $\mathrm{C}_{20} \mathrm{H}_{22} \mathrm{~N}_{2} \mathrm{O}_{5}$, together with its degraded ions at $m / e 332,266,234,228,213$ and 210, suggestive for the $\mathrm{N}$-salicylidene 2-DSA moiety. ${ }^{6)}$ A hexose fragment ion with $\mathrm{C}_{6} \mathrm{H}_{11} \mathrm{O}_{5}$ was weakly observed at $m / e$ 163. Fragment ions arising from the cleavage of a sugar ring appeared at $m / e 399,253$ and 238. Simitarly, MS of the N-salicylidene-O-TMS derivatives showed 
Fig. 3. TLC* of methanolyzates and hydrolyzates of SS-56 A,B,C and D (A-396-I) and related compounds

*Silica-gel $F_{254}$ TLC plate (E. Merck).

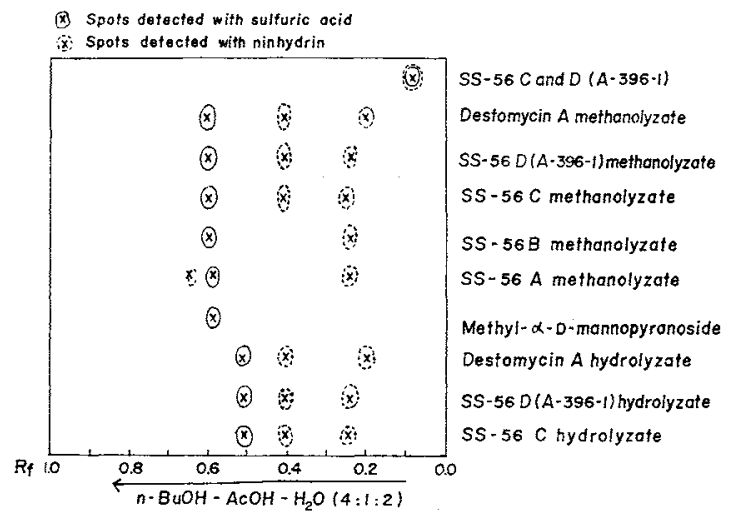

Fig. 5. GLC of hydrolyzates of SS-56 C V, TMS- $\alpha$-D-talose; VI, TMS- $\beta$-D-talose; TMS-streptamine; VII, TMS-destomic acid

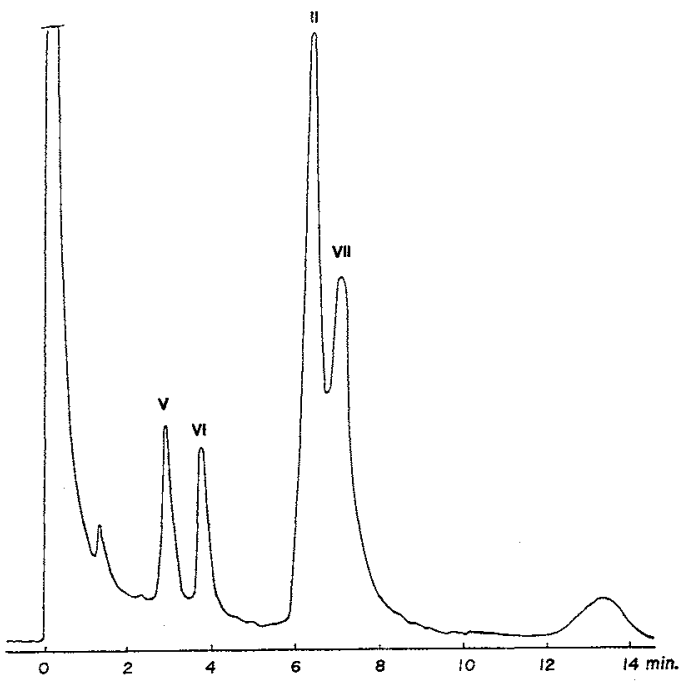

Fig. 4. GLC of methanolyzates of SS-56 C. I, TMS-methyl taloside; II, TMS-streptamine; III, IV, TMS-destomic acid, its methyl ester

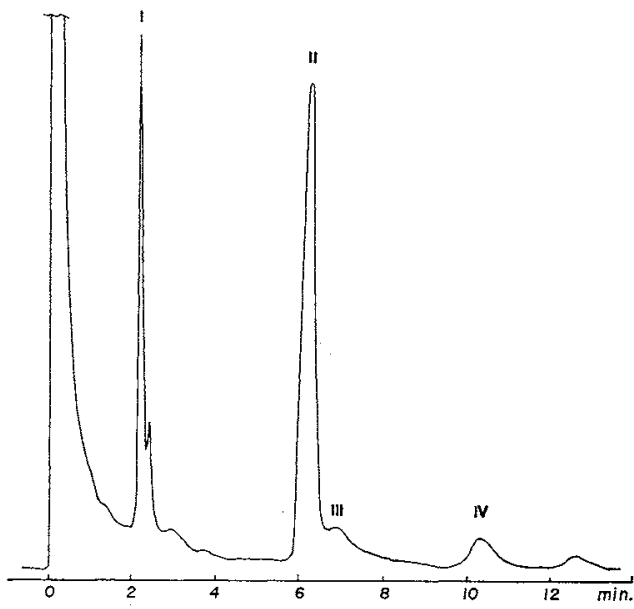

the $\mathrm{M}^{+}$at $m / e 1,108$, a 2-DSA fragment at $m / e 643$ and hexose fragments at $m / e 451$ and 363. Thus, the hexoglycosyl 2-DSA structure was suggested to be SS-56 A (5) and $B(6)$.

This was supported by the NMR spectra shown in Fig. 6. The spectra showed a quartet at 1.27 or 1.28 , and a double-triplet at 2.06, assignable to 2methylene protons of 2-DSA. A broad singlet appeared at 4.93 or 4.85 could be assigned to an anomeric proton of the hexose moiety.

Chemical degradation of 5 and $\mathbf{6}$ was summarized in Scheme 2. Methanolysis of 5 yielded crystalline 2-DSA dihydro-

chloride and amorphous methyl hexoside. The latter was identified as methyl $\alpha$-mannopyranoside by TLC (Fig. 3) and GLC (Table 5), and by conversion to crystalline methyl 2, 3, 4, 6-tetra-Oacetyl- $\alpha$-mannopyranoside.

Methanolysis of 6 gave crystalline 2-DSA dihydrochloride. Methyl hexoside recovered from the mother liquor showed identical behaviors on TLC (Fig. 3) and GLC (Table 5) as methyl taloside prepared from destomycin A and A-396-I. Combination of these results indicated that SS-56 A (5) is mannosyl 2-DSA and that SS-56 B (6) is talosyl 2-DSA.

Partial acid hydrolysis of SS-56 D (A-396-I) with N sulfuric acid, followed by chromatography over Dowex $1 \times 2\left(\mathrm{OH}^{-}\right)$resin with water as a developing solvent gave a crystalline compound, 
m.p. $230 \sim 231^{\circ} \mathrm{C}(\mathrm{dec})$. This was identical with SS-56 B with respects of Rf, IR, NMR and MS spectra and ORD curves. It was established therefore that SS-56 $\mathrm{B}$ has a partial structure of SS-56 D (A-396-I) coproduced as a main product.

We could not determine the ring form of mannoside and glycosidic position of 2-DSA in $\mathbf{5}$, but the structure $\mathbf{5}$ may be tentatively assigned to SS-56 A from the consideration of the biosynthetic relationship to other components (SS-56 B, $\mathrm{C}$ and $\mathrm{D})$. Indeed, negative $[\alpha]_{D}$ and negative ORD curves of SS-56 A and B were consistent with those of methyl $\beta-D$. manno or $\beta$-D-talopyranosides and 5-O-( $\beta$-D-talopyranosyl) hyosamine $(8)^{7}, 8$.

\section{Discussion}

The producing microorganisms of SS-56 C and A-396-I substances were both classified as $S$. eurocidicus, but components of antibiotics produced were considerably different.
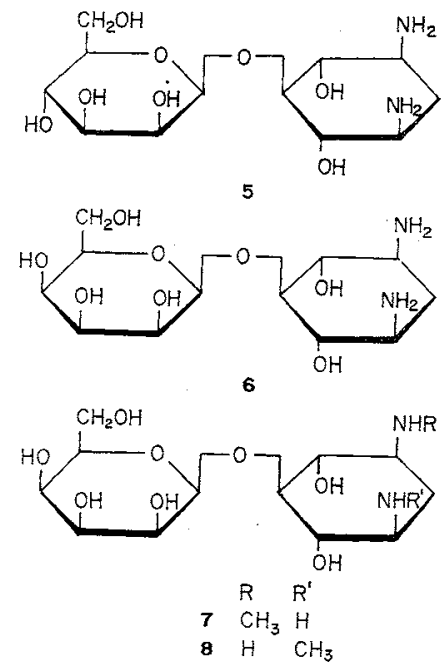

Chart 5. Suggested structures of SS-56 A (5), B (6), hygromycin $\mathrm{B}_{2}(7)$ and basic glycoside from destomycin $\mathbf{A}(\mathbf{8})$

Fig. 6. NMR spectra of SS-56 A (1) and $\mathrm{B}(2)$ in $\mathrm{D}_{2} \mathrm{O}$ at $70^{\circ} \mathrm{C}$

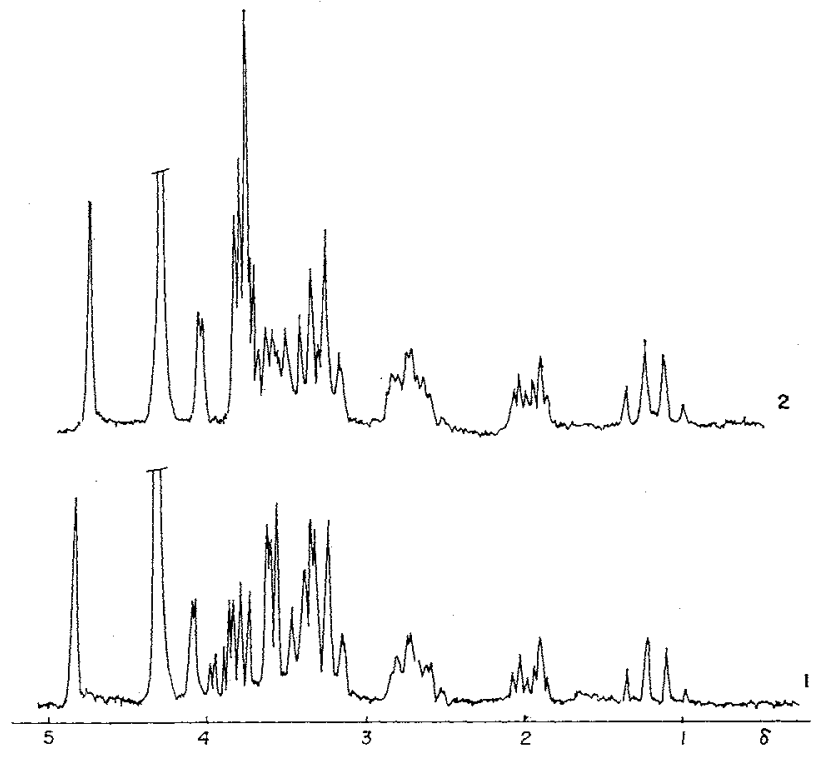

The strain isolated by SHoil et al. ${ }^{3)}$ was reported to produce A-396-I and hygromycin B in an equal ratio, whereas the strain of our laboratory produced A-396-I (SS-56 D) as a main component and SS-56 C as a minor. In spite of extensive search, no evidence was obtained on the formation of hygromycin B or destomycin A.

The isolation of two hexoglycosyl 2.DSA has not been reported in the past. Two hexoglyco. sides of $(+)$ or $(-)$ hyosamines (hygromycin $B_{2}(7)^{97}$ and basic glycoside from destomycin $\left.A(8)^{7}\right)$ were obtained by mild acid hydrolysis of the parent antibiotics. Although SS-56 B could be deriva. ble from SS-56 D by acid treatment, simultaneous isolation of two glycosides ( 5 and 6 ) from the 
broth under non-acidic condition indicated that they are not artifacts, but the true metabolites, implying the close relationship of SS-56 B and D in the biosynthesis. In this respects, particularly noteworthy was the isolation of mannosyl 2-DSA (5). Since mannoside contains $c i s-\alpha$-glycol at C-2 and C-3, just as taloside, it could be ortho-esterified with destomic acid to construct a new compound with similar molecular conformation to those of the destomycin group. Therefore, it would be interesting to see, if an antibiotic that contains $\beta$-D-mannose instead of $\beta$-D-talose of the destomycin group could be found in nature.

\section{Experimental}

General methods: NMR spectra were measured at $100 \mathrm{MHz}$ with a JNM-4H-100 spectrometer with DSS as an internal standard in $\mathrm{D}_{2} \mathrm{O}$. Chemical shifts were given on the $\delta$ scale. ORD curves were recorded with a JASCO Model ORD/UV-5 instrument at $25^{\circ} \mathrm{C}$, and IR spectra with a Hitachi Model 215 IR spectrophotometer in $\mathrm{KBr}$ tablet.

Trimethylsilylation and GLC: SS-56 A, B, C, D, neamine, A-396-I and destomycin A were trimethylsilylated by treating the respective free bases with TMS-PZ at $120^{\circ} \mathrm{C}$ for 10 minutes, and the resulting solution directly injected to a column at $230^{\circ} \mathrm{C}$ (SS-56 A, B and neamine) or at $310^{\circ} \mathrm{C}$ (SS-56 C, D, A-396-I and destomycin A). Streptamine, 2-epi-streptamine, methyl- $\alpha$-D-glucopyranoside and mannopyranoside were trimethylsilylated with TMS-PZ at $50^{\circ} \mathrm{C}$ for 15 minutes. TMS-Dglucose was supplied by Tokyo Kasei Kogyo, Tokyo. Methanolyzates and hydrolyzates in the form of hydrochloride were trimethylsilylated with a mixture of TMS-PZ and TMS-diethylamine $(2: 1)$ at $50^{\circ} \mathrm{C}$ for 30 minutes, and all injected to a column of $170^{\circ} \mathrm{C}$.

GLC was carried out using a Hewlett-Packard Gas Chromatograph Model 402 equipped with dual flame ionization detectors, and developing with helium at a flow rate of $c a 60 \mathrm{ml} / \mathrm{min}$. The column was of $0.4 \times 120 \mathrm{~cm}$ U-shaped glass tube, packed with $0.75 \% \mathrm{OV}-1$ on Gas-Chrom $\mathrm{Q}$.

Preparation of SCHIFF bases and their O-TMS derivatives: Four mg of each SS-56 A, B, C, D, and destomycin $A$ were dissolved in aqueous methanol $(1: 1)(0.2 \mathrm{ml})$, and excess of salicylaldehyde $(0.02 \mathrm{ml})$ was added. The mixture was stood at room temperature for 1 hour, and then evaporated to dryness in vacuo. Reprecipitation from iso-propanol-cyclohexane gave the respective SCHIFF bases of SS-56 A, m.p. $155 \sim 158^{\circ} \mathrm{C}$, SS-56 B, m.p. $146 \sim 149^{\circ} \mathrm{C}$, SS-56 C, m.p. $215 \sim 218^{\circ} \mathrm{C}$, SS-56 D, m.p. ca $200^{\circ} \mathrm{C}$ with sintering at $180 \sim 182^{\circ} \mathrm{C}$, and destomycin A, m.p. ca $200^{\circ} \mathrm{C}$ (decomp.) with sintering at $174 \sim 177^{\circ} \mathrm{C}$.

Treatment of the N-salicylidene SCHIFF bases with TMS-PZ at room temperature for 15 minutes gave the $\mathrm{N}$-salicylidene-O-TMS-derivatives, which were directly subjected to MS analysis without further purification.

MS spectra: Low-resolution MS spectra were recorded on a JMS-OISG double focussing mass spectrometer at $75 \mathrm{eV}$ using the electric recording and with resolution of 2,000 for the N-salicylidene. O-TMS derivatives. For the measurement of N-salicylidene SCHIFF bases, a resolution of 700 coupled with a maximum accelerating voltage $(8 \mathrm{KV})$ and a maximum ionizing current $(400 \mu \mathrm{A})$ were used to get a maximum gain. High resolution measurements were determined with a resolution of $c a 20,000$. The exact MS data were obtained by the photographic recording with PFK as an internal standard. The plates used were highly sensitive plates supplied from TECH/OPS, U.S.A. All values were within \pm 2 milli mass units below $m / e 400$, and \pm 5 milli mass units above $m / e 400$ of the theoretical values calculated for elemental compositions.

\section{Acknowledgements}

We are indebted to Dr. J. Shorr of Shionogi \& Co., Ltd. and Prof. T. SuAmi of Keio University for kindly providing samples of A-396-I substance and 2-epi-streptamine dihydrochloride, to Mr. KoDAMA for MS spectra, and to Miss SekiYA for elemental analysis. 


\section{References}

1) Kondo, S.; E. Akita \& M. Korke: The structure of destomycin A. J. Antibiotics, Ser. A 19: 139 140, 1966

2) Neuss, N.; K.F. Koch, B.B. Molloy, W. Day, L.L. Huckstep, D.E. Dorman \& J.D. Roberts: Structure of hygromycin B, an antibiotic from Streptomyces hygroscopicus; The use of CMR spectra in structure determination. I. Helv. Chim. Acta 53: 2314 2319, 1970

3) Shoj J.; S. Kozuki, M. Mayama, Y. Kawamura \& K. Matsumoto: Isolation of a new watersoluble basic antibiotic A-396-I. J. Antibiotics 23: $291 \sim 294,1970$

4) ShoJ, J. \& Y. Nakagawa: Structural feature of antibiotic A-396-I. J. Antibiotics 23: 569 571, 1970

5) Okami, Y.; R. Utahara, S. Nakamura \& H. Umezawa: On Streptomyces producing a new antifungal substance mediocidin and antifungal substances of fungicidin-rimocidin-chromin group, eurocidin group and trichomycin-ascosin-candicidin group. J. Antibiotics, Ser. A 7: 98 103, 1954

6) INOUYE, S.: Mass spectrometric analysis of aminoglycosidic antibiotics as N-salicylidene SCHIFF bases. Chem. Pharm. Bull. (Tokyo) 20: 2331 2347, 1972

7) Kondo, S.; M. Sezaki, M. Korke \& E. Akita: Destomycin A. The acid hydrolysis and the partial structure. J. Antibiotics, Ser. A 18: 192 194, 1965

8) Akita, E.: Optical rotatory dispersion of destomycin A and hygromycin B. Sci. Reports, Meiji Seika Kaisha 9: 65 66, 1967

9) Wiley, P.F.; M.U. Sigal, Jr. \& O. Weaver: Degradation products of hygromycin B. J. Org. Chem. 27: $2793 \sim 2796,1965$ 